effects of simple melt withdrawal, but the deposition of Ca-poor pyroxene leads the residue to be overly rich in silicon compared with that expected for melt withdrawal.

Anomalous Si enrichment in otherwise depleted mantle rocks is a common feature of the deep mantle samples brought to the surface from beneath continents by certain types of explosive volcanic eruptions. If the explanation proposed by Kelemen et al. for the anomalous $\mathrm{Si}$ and incompatible-element abundances of many of these samples of deep subcontinental mantle is correct, the evidence

\title{
CYTOKINES
}

\section{Can there be life without LIF?}

\section{John K. Heath}

LEUKAEMIA inhibitory factor (LIF) is a polypeptide growth factor with a seemingly remarkable range of biological actions in different tissue systems. From studies in tissue culture these include (in rough developmental sequence) the pluripotential stem cells of the early embryo, primordial germ cells, peripheral neurons, osteoblasts, adipocytes, hepatocytes and endothelial cells, as well as various leukaemic cells of the myeloid lineage ${ }^{1}$. Perhaps the most dramatic manifestations of the diverse action of LIF result from chronic administration of LIF to adult animals. These manifestations include rapid weight loss, behavioural disorders, ectopic calcification and bone abnormalities ${ }^{2}$.

If too much LIF is clearly bad for you, what are the consequences of having no LIF at all? The answer, rather unexpectedly, is remarkably little in respect of the known targets of LIF action, but quite a lot in terms of female reproductive physiology. On page 76 of this issue, Stewart et al. report that female mice harbouring genetically inactivated LIF genes have a specific defect in uterine function which prevents implantation of the developing embryo ${ }^{3}$. This result not only raises questions about the biological activities of polyfunctional cytokines like LIF but should lead into a better understanding (and ultimate control) of uterine function at the time of implantation.

Stewart et al. generated inactivating mutations in the gene encoding LIF using the techniques of gene targeting in embryonic stem cells ${ }^{3}$. Breeding chimaeras transmitting the mutant gene led to viable homozygous animals lacking functional LIF protein. These animals appear normal (aside from a small decrease in body mass) with no obvious signs of the anticipated dire phenotypic consequences of LIF deficiency. But, although adult males are fertile, adult

NATURE · VOL 359 - 3 SEPTEMBER 1992 suggests that much of the deep, stable mantle beneath continents has been modified chemically by the passage of melts. Thus, interaction with passing melts may be a process of comparable importance to partial melting and crystallization in magma chambers in determining the chemical composition of the continental crust and its underlying section of mantle.

Richard W. Carlson is in the Department of Terrestrial Magnetism, Carnegie Institution of Washington, 5241 Broad Branch Road NW, Washington, DC 20015, USA. result is how a growth factor can have so many functions that become obvious from its overexpression, and yet so few when tested by loss of function. It is becoming clearer as more and more genes succumb to gene targeting techniques that control system design in multicellular vertebrates is impressively sophisticated. It may be that many intercellular control systems have a powerful compensation facility by which the loss of one participant is redressed through altered action of another factor with analogous functions. In the case of LIF, a number of understudies are waiting in the wings: in many cases the actions of LIF in vitro can be reproduced by one or more of a select group of growth factors, which includes interleukin-6 and interleukin-11, oncostatin $\mathrm{M}$ and ciliary neurotrophic factor.

This capacity to swap functions has recently acquired a molecular foundation with the appreciation that at least interleukin-6, oncostatin M, ciliary neurotrophic factor and LIF share a common feature in their action: they all use the transmembrane glycoprotein 'signal converter' molecule gp130 as part of a mechanism to transduce growth factor binding to the outside of the cell into intracellular molecular signals ${ }^{5,6}$. The current data are consistent with a model for the action of this family of growth factors, in which association of the ligand with a 'private' ligand-specific receptor leads to the formation of a signalling complex including a 'public' class-specific transducer in the form of gp130.

In this model all members of a growth factor family sharing a common 'public' transducer can compensate for each other, providing the appropriate 'private' receptor chains are expressed. So whereas genetic inactivation of other members of the gp130 family of ligands might have specific, but relatively restricted, phenotypic consequences in vitro compared with their range of action in vitro, inactivation of gp130 would lead to widespread defects encompassing the combined cellular targets of the entire family. These models will doubtless shortly be tested experimentally, but in the meantime the LIF-less mouse reminds us that we can perhaps have too much of a good thing.

John $K$. Heath is in the Department of Biochemistry, University of Oxford, South Parks Road, Oxford OX1 3QU, UK. mouse ulthough the physiology of the of the human, it will be interesting to find out the extent to which LIF malfunction is involved in human reproductive failure and to see if implantation rates can be improved by administration of exogenous LIF over the implantation period.

A more general issue to arise from this
1. Polyfunctional Cytokines: $/ L-6$ and LIF Ciba Foundn Symp. 167 (Wiley, 1992).

2. Metcalf, D. \& Gearing, D. Proc, natn. Acad. Sci. U.S.A 86, 5948-5952 (1989).

3. Stewart, C. L. et al. Nature 359,76-79 (1992)

4. Bhatt, H., Brunet, L. \& Stewart, C. Proc. natn. Acad. Sci. Bhatt, H., Brunet, L. \& Stewart, C.
U.S.A. 88, 11408-11412 (1991).

5. Gearing, D. et al. Science 255, 1434-1437 (1992).

6. Ip. N. et al. Cell 69, 1121-1132 (1992). 